\title{
RESONANT PIEZOELECTRIC CANTILEVERS AS NANO-PRECISION DISPLACEMENT PROBES
}

\author{
M. Mahdavi $^{I^{*}}$, A. Abbasalipour ${ }^{1}$, A. Moses $^{2}$ and S. Pourkamali ${ }^{1,2}$ \\ ${ }^{1}$ The University of Texas at Dallas, Richardson, Texas, USA \\ ${ }^{2}$ femtoScale Inc., Denver, Colorado, USA
}

\begin{abstract}
This work presents a new class of highly sensitive displacement probes with sub-nm displacement resolution using a piezoelectric resonator as a frequency output strain gauge. The device is comprised of a length extensional bulk mode thin film piezoelectric on silicon (TPoS) resonator coupled to a V-shaped micro-cantilever. In this manner deflection of the cantilever tip is transferred to the resonating plate causing a change in its resonance frequency. Operating at $8.4 \mathrm{MHz}$, fabricated sensors show sensitivities as high as $1.5 \mathrm{~Hz} / \mathrm{nm}$ with a potential displacement resolution of $0.5 \AA$. The high sensitivity and fast response time along with a wide range of linearity make such probes suitable for different dynamic measurements in the sub-nm to a few microns range. Such devices can be used in compact fully electronic (non-optical) atomic force microscopes (AFM) or high resolution surface profilometry.
\end{abstract}

\section{INTRODUCTION}

Atomic force microscopes (AFMs) have proven to be invaluable tools in different state of the art micro and nanotechnology research areas with applications in high resolution surface imaging, high density data storage, biosensing and nanolithography [1-4]. At the heart of an AFM is a micro-cantilever that deflects as it scans a surface, typically due to atomic forces, and monitoring of its deflections can provide a high-resolution 3dimentional image of the surface. Different approaches have been sought to measure cantilever tip deflection. Current modulation of a tunneling tip was the first method initially used within the first AFMs to measure the cantilever deflections resulting from atomic forces on the surface of interest [1]. This method was capable of measuring forces in the $10^{-18} \mathrm{~N}$ range with displacement resolution of $\sim 1 \AA$. A few years later, optical levers were introduced providing unrivaled measurement resolution in the sub- $\AA$ range [5]. Although the optical lever is still the most common approach in commercially available scanning probe microscopes, complexity and bulkiness remain its main challenges, especially when measurements in adverse environments, such as low temperature or ultra-high vacuum conditions, are needed.

Electrically readable integrated force probes with nanometer precision are of great interest as potential replacements for bulky and complex optical levers. Chip-scale MEMS force probes could provide such powerful solutions with limited to no compromise in performance. Embedding piezoresistive strain sensors within microcantilevers is among popular techniques showing sub-nm resolution [2], [6]. Piezoelectric detection is another method explored to measure induced stress along the cantilever due to deflection [7]. However both of such displacement/force measurements happen at DC which necessitates low bandwidth measurement in order to reduce interference of low frequency noise. This constraint in turn limits the response time of the probe. Moreover such techniques require a high precision $\mathrm{A} / \mathrm{D}$ conversion circuit to measure minuscule voltage or current changes resulting from the week transduction mechanisms. In addition to above-mentioned disadvantages, a piezoresistive strain gauge requires a relatively high DC bias which also causes higher power dissipation and consequently temperature instability [2], [6].
On the contrary, frequency-modulated output at higher frequencies provides a faster response, and is less vulnerable to noise, particularly low frequency noise of the DC measurements. Furthermore, frequency measurements can be performed directly by a digital frequency counter without the need for A/D conversion.

Micro-fabricated capacitive and piezoelectric displacement sensors for frequency output tapping mode microscopy have been reported [8], [9]. Although resolving issues of previous amplitude measurements, such methods require operation in vacuum to avoid performance deterioration due to air damping while resonating at flexural resonance mode. Moreover, capacitive detection needs a small gap and relatively high bias voltage to achieve high displacement sensitivity.

Thin film piezoelectric-on-substrate (TPoS) resonators have been used for a wide range of sensory applications such as high resolution mass sensing [10], and pressure and temperature measurement [11], [12]. Such lateral extensional mode resonant devices can be used in low power self-sustain oscillating circuits providing highly stable frequency sources without operation in vacuum. In this work, for the first time, a TPoS resonator has been coupled to a cantilever. Bending of the cantilever deforms the resonator changing its resonance frequency. Such design can lead to a high throughput displacement probe addressing deficiencies of prior microscale detection mechanisms. Piezoelectric transduction can also bypass temperature stability issues resulting from selfheating (due to the required DC bias) of piezoresistive readout.

\section{OPERATION PRINCIPLES}

The proposed displacement probe consists of a rectangular BAW resonator with two triangular cantilever extensions on both ends. The resonator is used as a frequency output strain gauge operating in its first extensional resonance mode as illustrated in Figure 1(a). A piezoelectric transducer was used in order to actuate such resonance, which is made of a $1 \mu \mathrm{m}$ thick AlN layer sandwiched between two platinum electrodes.

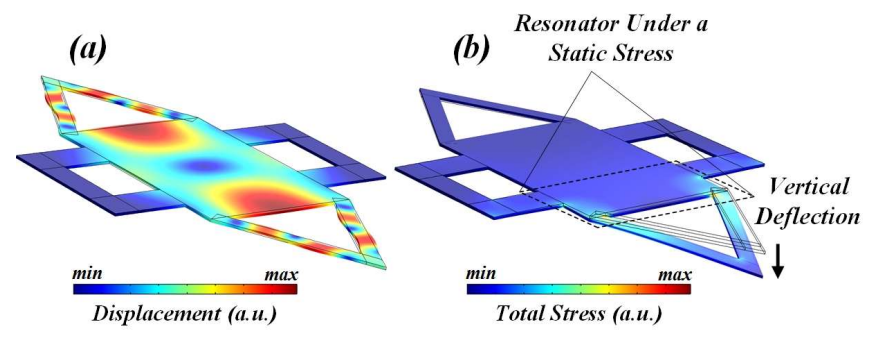

Figure 1: (a) 8.4MHz first length extensional resonance mode of the resonator coupled with $V$-shaped cantilevers, (b) static mechanical stress on the cantilever and the resonator resulting from a static force applied to the cantilever tip.

Figure 1(b) shows the stress on the cantilever and the resonator resulting from a static force applied to the tip of the V-shaped cantilever. The mechanical stress modulates the resonance frequency of the resonator. V-shaped cantilevers are added on both 
sides and at the corners of the resonator to maintain symmetry for the resonant structure.

A typical AFM cantilever has a spring constant $(K)$ in the range of 0.01-100 N/m. Depending on the type of microscopy, there is a trade-off between force and displacement sensitivity; a stiffer probe provides a higher displacement sensitivity, while a softer probe provides better force sensitivity. However, low stiffness probes are required for contact AFM to avoid surface damage. Moreover, fabricating the cantilever and resonator from the same silicon device layer, an additional compromise exists between quality factor $(Q)$ of the resonator and stiffness of the cantilever in the design of the proposed structure. A thicker silicon substrate leads to a higher resonator $Q$, which improves the frequency stability of the TPoS resonator when engaged in an oscillating circuit configuration. In contrast, stiffness is proportional the substrate thickness cubed. Probe stiffness can also be reduced by increasing the length of the cantilever instead of reducing the thickness. Nevertheless, a longer cantilever can potentially disturb the operation of the resonator, and reduce the effective stress resulting from deflection on the resonator, thus decreasing the displacement sensitivity.

On the other hand, the performance of a resonator can be evaluated based on its motional resistance $\left(R_{m}\right)$ [13]. In order to engage the resonator in a single stage self-sustained oscillating circuit, $R_{m}$ needs to be minimized. The electrode area on the resonator is inversely proportional to the motional resistance. The length of the resonator which determines the resonance frequency is not easily adjustable, and therefore the lateral surface area only can be controlled by the resonator width. Increasing the width of the resonator increases the probe stiffness while decreasing the motional resistance simultaneously. Therefore, a trade-off between the spring constant of the probe and the motional resistance of the resonator should be addressed with a proper selection of lateral surface area.

The structure was fabricated on a $5 \mu \mathrm{m}$ silicon device layer. Such thickness has been chosen in order to provide a fair performance of the structure as a force/displacement probe and reliable frequency source component at the same time. The stiffness of the triangular cantilever alone with the length of $330 \mu \mathrm{m}$ was found to be $16.8 \mathrm{~N} / \mathrm{m}$ based on the static force simulation results. After coupling with the resonator, overall probe stiffness was found to be $9.2 \mathrm{~N} / \mathrm{m}$. The resonator horizontal dimensions are chosen to be $420 \times 315 \mu \mathrm{m}^{2}$ leading to first lateral resonance mode frequency of $8.4 \mathrm{MHz}$ and motional resistance for the resonator that is low enough for operation in a single stage oscillation circuit.

\section{EXPERIMENTAL AND METHOD}

\section{Fabrication Process}

The resonant piezoelectric probes were fabricated on a SOI substrate with $5 \mu \mathrm{m}$ thick $<100>$ oriented low resistivity device layer. Figure 2 shows the fabrication process flow. Bottom electrodes patterns were first formed on the substrate with negative nLOF2020 photoresist. A $150 \mathrm{~nm}$ of Pt layer forming the bottom electrodes was then sputtered and lifted-off. A $1 \mu \mathrm{m}$ AlN piezoelectric layer was then deposited (Figure 2(a)) and patterned to provide electrical accesses to the bottom electrode. AlN etch was performed in two steps: a chlorine-based ICP etch to remove the main bulk of the $2 \mu \mathrm{m}$ thick layer followed by a wet-etch step in $2.5 \%$ TMAH to remove the remainder of the AlN layer. Similar to the bottom metal electrodes, top platinum electrodes (150nm thick) were formed via sputtering and lift-off (Figure 2(b)). Later a $1.5 \mu \mathrm{m}$ layer of low temperature LPCVD oxide was deposited to be used as a hard mask for the upcoming silicon etch step and to protect the
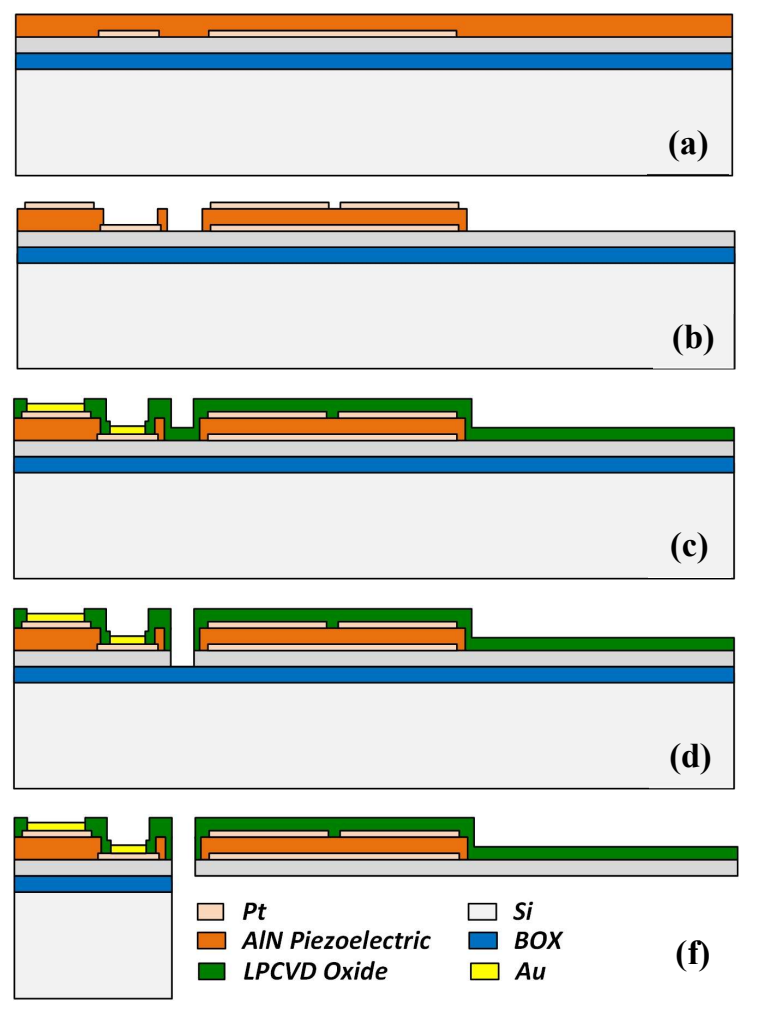

Figure 2: Fabrication process of resonant piezoelectric cantilevers.

AlN layer during etching and cleaning steps. An 800nm gold layer was deposited and lifted-off on wire-bond pads after etching the oxide layer on the pad areas (Figure 2(c)). After etching the oxide hard mask layer with plasma, silicon was etched via DRIE to form the resonator and cantilever out of the device layer (Figure 2(d)). Finally, to suspend the whole structure, silicon handle-layer and BOX layer were etched via silicon DRIE and fluorine based ICP respectively from backside (Figure 2(f)).

The SEM view of fabricated probe is illustrated in Figure 3. The cantilever arms are merely made of the same silicon device layer. Furthermore, the top platinum layer is divided into actuation and sensing electrodes allowing two-port operation of the resonator.

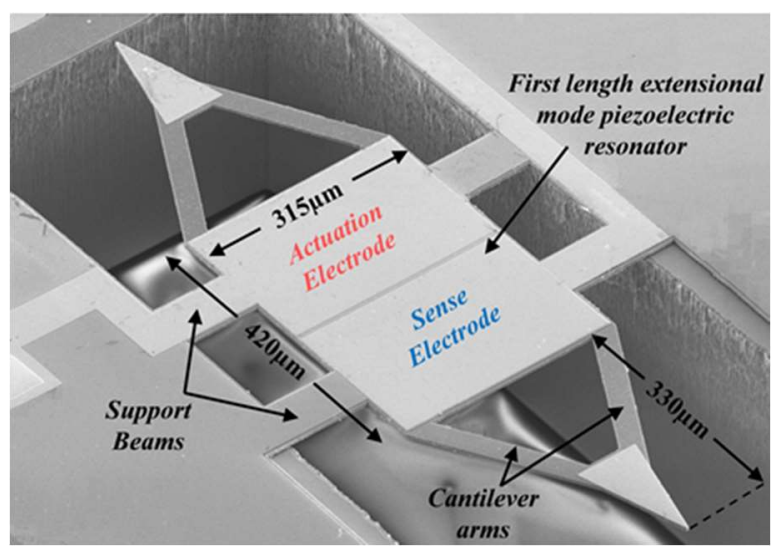

Figure 3: SEM image of a fabricated resonant piezoelectric cantilever comprised of a first length extensional mode thin film piezoelectric-on-substrate (TPOS) resonator with triangular cantilever extensions. 


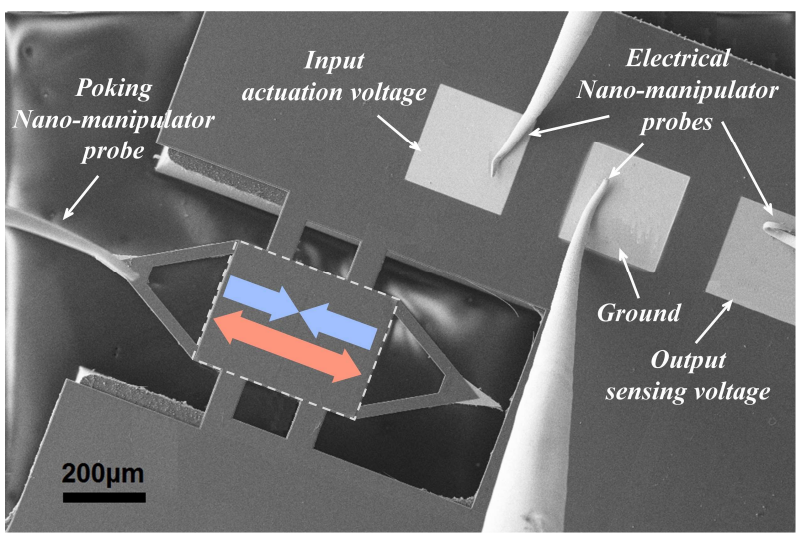

\section{Characterization}

In-situ SEM characterization of the displacement sensors was performed inside a scanning electron microscope (SEM) using a Zyvex S100 Nano-manipulator as shown in Figure 4(a). Input, output and ground electrical connections from network analyzer to the device inside the SEM chamber were provided using three nanomanipulator probes while a forth probe was used to deflect the cantilever. The working distance (WD) of the SEM electromagnetic lens was used to measure the displacement at the cantilever tip (Fig. $3 b)$. The WDs were read at high magnification to maximize reading accuracy. Figure 5 demonstrates the measured frequency responses of the resonator under different cantilever tip displacements. The resonator has a resonance frequency of $8.4 \mathrm{MHz}$ with a quality factor of $\sim 900$ and motional resistance of $3 \mathrm{k} \Omega$. Frequency shifts resulting from four different displacements at the free end of the probe are evident in acquired frequency responses. Resonance peaks suggest that deformation resulting from higher deflection of the cantilever slightly reduces the peak amplitude and quality factor due to deformation of the resonator. Device characterization does not show a noticeable change in $Q$ resonator in air and under vacuum.

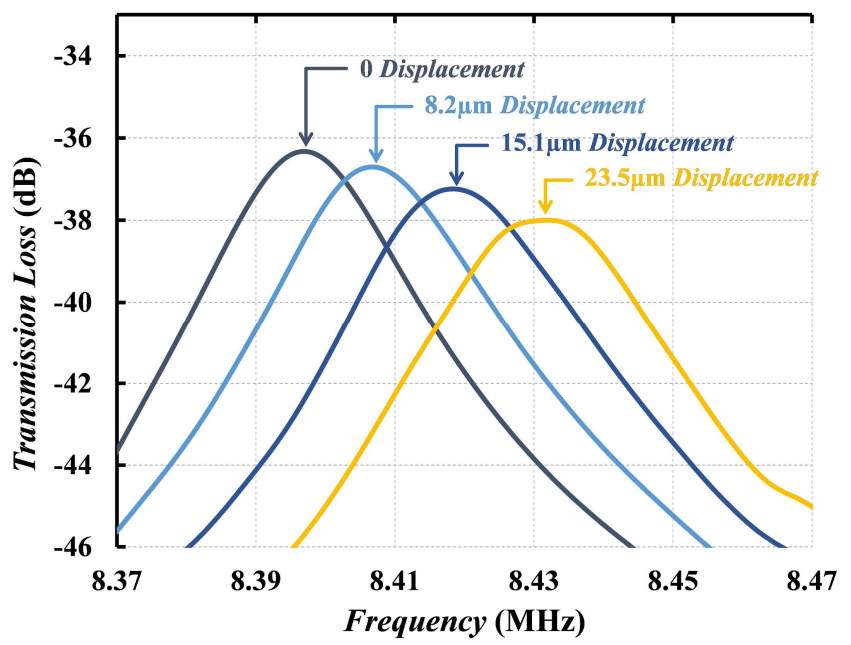

Figure 5: The resonance frequency of the device changes in response to vertical displacement of cantilever tip.

Figure 6 graphs the measured resonator frequency shifts versus probe tip deflection showing a linear response with sensitivity of $\sim 1.5 \mathrm{~Hz} / \mathrm{nm}$ for a relatively wide $30 \mu \mathrm{m}$ range of displacements. The minimum detectable displacement (MDD) of the displacement probe is limited with frequency stability of the resonator. Engaging such resonator in a self-sustained oscillator circuit leads to $10^{-8} d f \mid f_{0}$

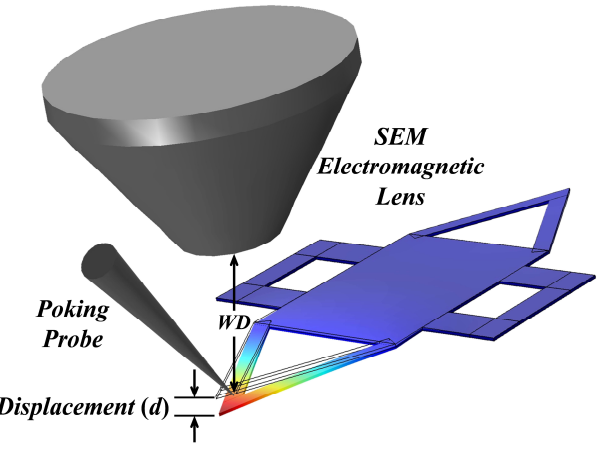

Figure 4: (a) InSEM

characterization of the displacement probe using nanomanipulator, working distance (WD) was used as a measure of displacement posed by the nanomanipulator probe.

Allan deviation in a 1 minute measurement period with sampling rate of $10 \mathrm{~Hz}$. Such frequency stability is associated with $0.08 \mathrm{~Hz}$ translating to a potential displacement resolution as small as $0.5 \AA$ for the fabricated probe. Considering Cantilever stiffness of $9.2 \mathrm{~N} / \mathrm{m}$ obtained from COMSOL finite element analysis a force resolution of $400 \mathrm{pN}$ has been achieve for such structure with a $5 \mu \mathrm{m}$ thick silicon device layer. Such pico-range force detection limit demonstrates a resolution force sensitivity of the probe along with its high resolution displacement sensitivity.

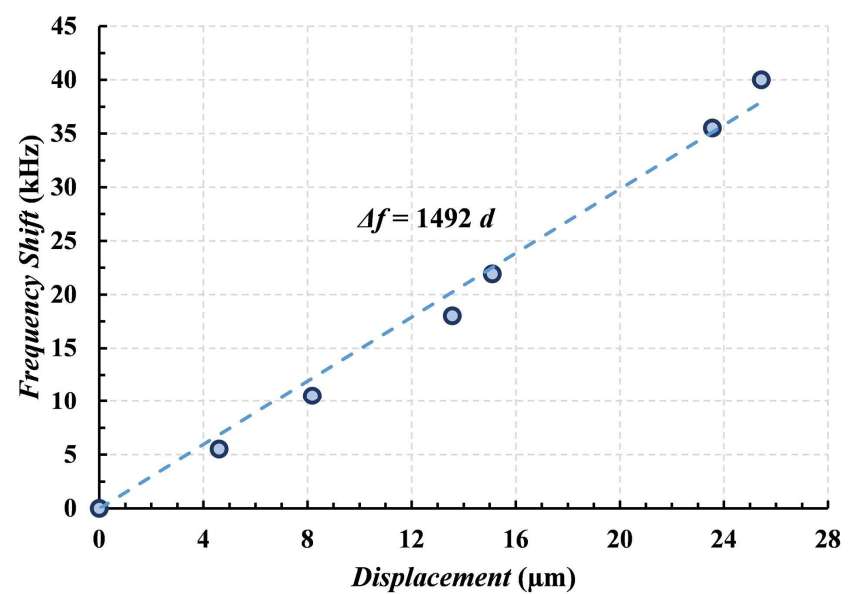

Figure 6: Resonance frequency shift vs. displacement at the end of cantilever

COMSOL analysis shows that frequency shift is not a function of device/cantilever thickness and therefore the device can be thinned down to increase force sensitivity by orders of magnitude while maintaining the same displacement sensitivity.

Different microscale displacement detection techniques as AFM probes are summarized in Table 1. The proposed displacement probe shows the minimum detectable displacement (MDD) in the same range compared to other approaches. However the current detection approaches do not provide versatile and robust schemes in microscale implementation of AFM probes.

\section{CONCLUSION}

A thin film piezoelectric-on-insulator (TPoS) MEMS resonator, vibrating at its first length extensional mode, was used as a frequency output strain gauge coupled with triangular cantilevers. Such structure was used as a displacement sensor showing linear sensitivity of $1.5 \mathrm{~Hz} / \mathrm{nm}$ over a wide range of cantilever tip 
Table 1: MEMS displacement probes comparison

\begin{tabular}{|c|c|c|c|c|}
\hline & $\begin{array}{l}\text { Detection Mechanism/ } \\
\text { Output }\end{array}$ & $M D D^{+}$ & Operation Mode & Disadvantages \\
\hline$[2]$ & $\begin{array}{l}\text { Piezoresistive Output/ } \\
\text { Voltage Amplitude }\end{array}$ & $\begin{array}{c}0.5 \AA \text { in } \\
10 \mathrm{~Hz}-1 \mathrm{kHz} \mathrm{BW}\end{array}$ & DC measurement & $\begin{array}{l}\text { Low frequency noises - Thermal instability - } \\
\text { Speed limit }\end{array}$ \\
\hline$[7]$ & $\begin{array}{l}\text { Piezoelectric Output/ } \\
\text { Current Amplitude }\end{array}$ & $\begin{array}{l}0.1-0.3 \AA \text { in } \\
125 \mathrm{~Hz} \mathrm{BW}\end{array}$ & DC measurement & Low frequency noise sources - Speed limit \\
\hline$[8]$ & $\begin{array}{l}\text { Capacitive Sensing/ } \\
\text { Frequency }\end{array}$ & $\begin{array}{l}0.5 \AA \text { ( } 2 \mathrm{~Hz} \text { freq. } \\
\text { stability) }\end{array}$ & $\begin{array}{l}\text { Flexural resonance } \\
\text { mode }-66.5 \mathrm{kHz}\end{array}$ & Small gap - Large DC bias - Air Damping \\
\hline [9] & $\begin{array}{c}\text { Piezoelectric } \\
\text { Output/Frequency }\end{array}$ & $\begin{array}{l}1 \AA \text { ( } 2 \mathrm{~Hz} \text { freq. } \\
\text { stability) }\end{array}$ & $\begin{array}{l}\text { Flexural resonance } \\
\text { mode }-95 \mathrm{kHz}\end{array}$ & Air Damping - Stiff cantilever \\
\hline $\begin{array}{l}\text { This } \\
\text { work }\end{array}$ & $\begin{array}{l}\text { Resonant TPoS/ } \\
\text { Frequency }\end{array}$ & $\begin{array}{l}0.5 \AA \text { (Freq. stability } \\
\text { of } 10^{-2} \mathrm{ppm} \text { ) }\end{array}$ & $\begin{array}{l}\text { Resonance at } 1 \text { st length } \\
\text { extensional }-8.4 \mathrm{MHz}\end{array}$ & \\
\hline
\end{tabular}

+ MDD: Minimum Detectable Displacement

deflections. The frequency stability of the resonator within the proposed probe demonstrates a potential minimum detectable deflection (MDD) of $0.5 \AA$. This detection limit is comparable with its state of the art counterparts without putting limits on scanning speed of the probe. Such frequency output displacement probes can also address problems associated with some of the previously demonstrated microscale detection mechanisms such as vulnerability to low frequency noise sources, temperature instability and vacuum operation.

\section{ACKNOWLEDGMENT}

Travel support has been generously provided by the Transducer Research Foundation. The project was supported under DOE funding of phase I SBIR. Authors would like to thank staffs of cleanroom at University of Texas at Dallas, especially Dr Roger Robbins for his help on SEM in-situ characterization.

\section{REFERENCES}

[1] G. Binnig, C. F. Quate, and C. Gerber, "Atomic Force Microscope," Phys. Rev. Lett., vol. 56, no. 9, pp. 930-933, Mar. 1986.

[2] B. W. Chui, T. D. Stowe, Y. S. Ju, K. E. Goodson, T. W. Kenny, H. J. Mamin, B. D. Terris, R. P. Ried, and D. Rugar, "Low-stiffness silicon cantilevers with integrated heaters and piezoresistive sensors for high-density AFM thermomechanical data storage," J. Microelectromechanical Syst., vol. 7, no. 1, pp. 69-78, Mar. 1998.

[3] N. Jalili and K. Laxminarayana, "A review of atomic force microscopy imaging systems: application to molecular metrology and biological sciences," Mechatronics, vol. 14, no. 8, pp. 907-945, Oct. 2004.

[4] R. D. Piner, J. Zhu, F. Xu, S. Hong, and C. A. Mirkin, "'DipPen' Nanolithography," Science, vol. 283, no. 5402, pp. 661663, 1999.

[5] G. Meyer and N. M. Amer, "Novel optical approach to atomic force microscopy," Appl. Phys. Lett., vol. 53, no. 12, pp. 10451047, Sep. 1988.

[6] J. Thaysen, A. Boisen, O. Hansen, and S. Bouwstra, "Atomic force microscopy probe with piezoresistive read-out and a highly symmetrical Wheatstone bridge arrangement," Sens. Actuators Phys., vol. 83, no. 1-3, pp. 47-53, May 2000.

[7] B. Rogers, L. Manning, T. Sulchek, and J. D. Adams, "Improving tapping mode atomic force microscopy with piezoelectric cantilevers," Ultramicroscopy, vol. 100, no. 3-4, pp. 267-276, Aug. 2004.

[8] N. Blanc, J. Brugger, N. F. de Rooij, and U. Dürig, "Scanning force microscopy in the dynamic mode using microfabricated capacitive sensors," J. Vac. Sci. Technol. B, vol. 14, no. 2, pp 901-905, Mar. 1996.

[9] Y. Miyahara, M. Deschler, T. Fujii, S. Watanabe, and H. Bleuler, "Non-contact atomic force microscope with a PZT cantilever used for deflection sensing, direct oscillation and feedback actuation," Appl. Surf. Sci., vol. 188, no. 3-4, pp. 450-455, Mar. 2002.

[10] M. Mahdavi, G. Guerra, H. McCurry, S. Pourkamali, and R. Abdolvand, "Piezoelectric resonant MEMS balances with high liquid phase Q," in 2014 IEEE SENSORS, 2014, pp. 19481951.

[11] R. Tabrizian and F. Ayazi, "Dual-mode vertical membrane resonant pressure sensor," in 2014 IEEE 27th International Conference on Micro Electro Mechanical Systems (MEMS), 2014, pp. 120-123.

[12] J. L. Fu, R. Tabrizian, and F. Ayazi, "Dual-Mode AlN-onSilicon Micromechanical Resonators for Temperature Sensing," IEEE Trans. Electron Devices, vol. 61, no. 2, pp. 591-597, Feb. 2014.

[13] G. K. Ho, R. Abdolvand, A. Sivapurapu, S. Humad, and F. Ayazi, "Piezoelectric-on-Silicon Lateral Bulk Acoustic Wave Micromechanical Resonators," J. Microelectromechanical Syst., vol. 17, no. 2, pp. 512-520, Apr. 2008.

\section{CONTACT}

*M. Mahdavi, tel: +1-469-360-6450; mmahdavi@utdallas.edu 